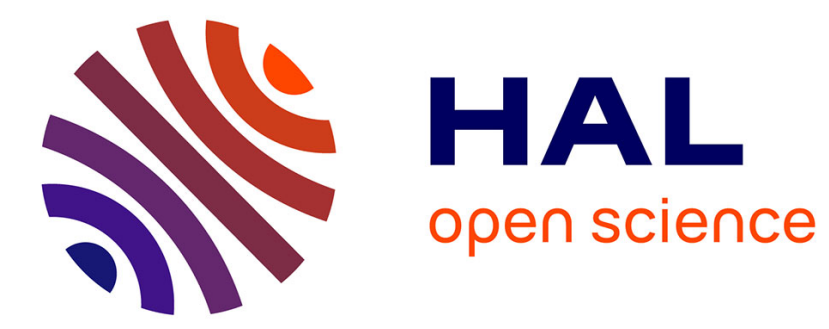

\title{
Habitants et architectes : des créateurs d'images
}

Valérie Lebois

\section{To cite this version:}

Valérie Lebois. Habitants et architectes : des créateurs d'images. Labyrinthe. Atelier interdisciplinaire, 2003, 15, 10.4000/labyrinthe.470 . hal-03464248

\section{HAL Id: hal-03464248 \\ https://hal.science/hal-03464248}

Submitted on 3 Dec 2021

HAL is a multi-disciplinary open access archive for the deposit and dissemination of scientific research documents, whether they are published or not. The documents may come from teaching and research institutions in France or abroad, or from public or private research centers.
L'archive ouverte pluridisciplinaire HAL, est destinée au dépôt et à la diffusion de documents scientifiques de niveau recherche, publiés ou non, émanant des établissements d'enseignement et de recherche français ou étrangers, des laboratoires publics ou privés. 


\section{Labyrinthe}

15 | 2003

Territoires : questions d'images

\section{Habitants et architectes : des créateurs d'images}

\section{Valérie Lebois}

\section{(2) OpenEdition}

Journals

Édition électronique

URL : http://journals.openedition.org/labyrinthe/470

DOI : $10.4000 /$ labyrinthe.470

ISSN : 1950-6031

Éditeur

Hermann

Édition imprimée

Date de publication : 1 juin 2003

\section{Référence électronique}

Valérie Lebois, « Habitants et architectes : des créateurs d'images », Labyrinthe [En ligne], 15 | 2003,

mis en ligne le 24 juillet 2008, consulté le 20 avril 2019. URL : http://journals.openedition.org/

labyrinthe/470 ; DOI : 10.4000/labyrinthe.470

Ce document a été généré automatiquement le 20 avril 2019.

Propriété intellectuelle 


\title{
Habitants et architectes : des créateurs d'images
}

\author{
Valérie Lebois
}

Images de nos territoires? Pour certains, la question ne mérite pas que l'on s'y attarde. Prenons, parmi eux, le cas de cet architecte qui, soucieux de produire dans un colloque une réflexion sociologique, avait superposé à ses projets de réhabilitation d'un grand ensemble des images de nids d'oiseaux et de terriers de lapins. Et de les commenter: "Tout être a besoin d'un abri; le tout est de lui trouver une forme adéquate.» S'approprier un territoire ne serait donc que la réponse à une fonction vitale, comme se protéger des risques naturels et des violences d'autrui. Pourtant, de nombreux travaux en sciences humaines se sont attachés à montrer que les liens qui se nouent entre les individus et leurs lieux de vie ne sont pas réductibles aux seuls rapports matériels ou fonctionnels. Ils incluent une valorisation qui déborde leur caractère physique et produit cet ensemble de significations symboliques à l'œuvre dans nos représentations mentales. Pour le tester sans détour, il nous suffirait d'évoquer deux types de territoires très contrastés, à la fonction pourtant similaire ${ }^{1}$ : d'une part, les vastes étendues caractérisant les pieds des grands ensembles et, d'autre part, les cours des immeubles parisiens pour constater qu'un album d'images défile dans nos têtes, chacun ne manquant pas de connoter avec force ces deux formes urbaines. Mais fi de démonstration à l'emportepièce, développons notre propos.

\section{PARLER D'IMAGE, POURQUOI ?}

\section{Habiter : faire son nid et un peu plus...}

Dès lors que des espaces sont occupés, ils sont transformés en lieux habités, des lieux qui se chargent d'histoires individuelles et collectives. "Ce sont des lieux « habités", des lieux de pratiques et de relations, des lieux de mémoire et des lieux de projets, des lieux imaginés aussi, chacun construisant et reconstruisant imaginairement des espaces 
fréquentés de manière singulière et par ailleurs partagés ${ }^{2}$. A Aussi, le territoire, dans sa capacité à imprimer les souvenirs et les symboles, joue un rôle social, car il devient matière à communication entre les individus. Il peut être assimilé «à un vaste système mnémonique pour la fixation des idéaux et de l'histoire du groupe ${ }^{3}$ ». Derrière cet investissement, autrement plus complexe que la recherche d'un seul confort matériel, se joue principalement une quête d'identité. Il s'agit tout à la fois de "configurer » son environnement, de lui reconnaitre des formes distinctes et uniques et, dans le même temps, de se forger une identité personnelle dans le but d'assurer sa participation au monde, à la communauté. En d'autres termes, un environnement m'est familier si je le reconnais et si je me reconnais à travers lui. Sous cet angle, l'appropriation se révèle comme un processus essentiellement psychique, «qui consiste à produire de la diversité en investissant l'espace d'intentions et d'actes qui permettent à l'individu de survivre à la banalité du quotidien et de se donner une identité, c'est-à-dire de créer des situations où l'espace constitue pour lui une ressource ${ }^{4} »$. Aussi, les conduites de l'habiter ne peuvent se penser sans considérer "l'investissement psycho-affectif qu'elles sous-tendent, investissement qui assure l'étayage - c'est-à-dire la construction et le ressourcement - de l'identité individuelle et sociale ${ }^{5}$ ». Nous considérerons alors l'appropriation territoriale, non comme une seule nécessité physique, mais bien comme la définit Gilles Barbey, «la reconquête permanente d'un espace physique banal sous la forme d'un lieu mental, chargé de sens ${ }^{6} »$.

3 S'intéresser aux images de nos territoires revient alors à considérer ces mécanismes de passage à l'abstraction et invite par là-même à se pencher sur les reconstructions mentales qui se produisent lorsqu'une réalité est perçue.

\section{Le filtre de l'image}

Penser que nous appréhendons notre environnement en pur observateur serait négliger la part de subjectivité qui se mêle inévitablement à notre perception. Ce que nous retenons de nos territoires est irrémédiablement modelé en fonction de notre pratique, de nos évaluations, de nos impressions et des significations que nous lui attribuons. En d'autres termes, nous connaissons nos environnements à travers les images que nous nous en faisons.

C'est pourquoi Kevin Lynch, chercheur célèbre pour ses travaux sur l'image de la cité insistait sur ce "processus de va-et-vient » qui se joue entre le sujet et l'objet observé : «Ce que le sujet voit est basé sur la forme extérieure de l'objet, mais la façon dont il l'interprète et l'organise et l'endroit où il porte son attention influe à son tour sur ce qu'il voit ${ }^{8}$.» Nos expériences perceptives sont donc stockées sous la forme de représentations mentales qui se distinguent de la réalité parleur caractère schématique, lacunaire, déformé, et souvent dominé par des facteurs affectifs. On comprend ainsi comment les lieux habités deviennent le support d'images multiples. Il y a notamment celles dont parle Bachelard, qui se forment dans l'enfance, dans ce premier milieu composé de rapports et d'espaces affectifs et qui orientent nos rapports aux espaces de toutes nos habitations futures. Et aussi toutes les images qui, de façon permanente, «se dédoublent des perceptions quotidiennes ou moins ordinaires, et mémorisées, recomposées, accompagnent la trame des jours et nourrissent la manière dont chacun rêve d'un ailleurs à partir d'un ici9. » 


\section{L'imagibilité ou le fort pouvoir évocateur de l'espace}

6 L'investissement par lequel l'individu se saisit de son environnement le fait exister, lui donne du sens, dépend de la personne elle-même, de ses attentes, de sa capacité à inscrire et à construire du sens, mais aussi des caractéristiques de l'environnement lui-même. C'est dans ce sens que Kevin Lynch, en cherchant à rendre compte de l'image globale de la ville qui se forme au fil du temps dans la représentation des citadins, s'interroge sur la capacité des éléments urbains à provoquer une forte image chez l'individu. Aussi traduitil cette qualité de l'objet physique à se distinguer et à se présenter aux sens d'une manière aiguë et intense par la notion d'imagibilité. C'est cette propriété que nous entendons questionner dans le rapport qu'entretiennent les individus à l'architecture : comment l'architecture contribue-t-elle à façonner des représentations individuelles ou sociales? comment participe-t-elle au développement ou aux mutilations de l'imaginaire? comment peut-elle susciter émotions et sentiments? Il faut entendre ici l'architecture non comme un objet visuel à regarder, mais bien comme « un véritable milieu de vie enclos dans les murs, un milieu de vie stimulant les sens. Ce sont des ombres et des lumières évidemment, des proportions et des couleurs, des perspectives et des décors, mais aussi des sons qui se réverbèrent, des surfaces que nos pieds foulent, des textures que l'on touche, des températures qui nous mettent à l'aise et des odeurs qui nous enveloppent et nous séduisent ${ }^{10}$.» Cette précaution nous conduit à ne pas négliger les données sensorielles autres que visuelles qui peuvent tout autant produire des images fortes, telles que des images sonores, tactiles.

7 Il nous semble d'ailleurs utile de repérer les différentes sources dans lesquelles puise notre capacité imageante.

\section{Les sources de nos images}

8 Comme nous l'avons déjà souligné, l'image d'un lieu est largement déterminée par la manière de le pratiquer, par les rencontres que l'on y fait, mais aussi à travers « l'expérience sensible » que l'on y vit. Chacun « éprouve » son rapport à l'espace. La mise en contact de notre corps avec les éléments extérieurs est instantanément génératrice de sensations multiples, liées aux odeurs, aux sons, aux textures, aux couleurs, aux formes... Par le filtre de cette activité sensorimotrice, les caractères concrets de l'environnement (grand, clair, silencieux...) se voient convertis en concepts psychologiques (confortable, sécurisant, reposant...) et deviennent alors déterminants dans l'évaluation et le souvenir de l'ambiance du lieu. Il n'est pas non plus inutile de rappeler que ces émotions éprouvées dépendent de notre état d'esprit. C'est ainsi que l'on peut parler de projection ${ }^{11}$ pour illustrer le processus à travers lequel nous nous représentons le milieu ambiant, et le valorisons positivement ou négativement en fonction de nos propres émotions, valeurs, habitudes ou attentes.

9 Cependant, les habitudes perceptives et visuelles ne sont pas seulement singulières, elles s'insèrent aussi dans un imaginaire commun. Comme le soulignait Kevin Lynch, «si chaque individu crée et porte en lui sa propre image, il semble qu'il y ait une grande concordance entre les membres d'un même groupe ${ }^{12} »$. De fait, les représentations se constituent à partir de nos expériences mais aussi à travers les modèles de pensée reçus et transmis par la tradition, l'éducation et la mémoire sociale. La signification des lieux 
résulte en effet des valeurs créées par la société, ce qui revient aussi à souligner l'importance de la charge culturelle inhérente à tout aménagement humain. De plus, les productions littéraires, artistiques, ne sont pas sans effet sur la formation de nos représentations. Les romanciers, les peintres, les photographes, les cinéastes, les sculpteurs construisent des images qui font partie elles aussi de notre société, imprègnent la perception des espaces que nous habitons. Il en est de même de l'environnement médiatique qui influe sur les représentations que tous les citadins ont de la ville.

\section{IMAGES DE NOS ESPACES D'À CÔTÉ : DEUX CAS DE FIGURE} lieux particuliers, très évocateurs chacun à leur manière dans le système urbain. On pourrait dire que les premiers portent les stigmates d'un urbanisme aujourd'hui durement condamné. Nous voulons parler de ces espaces qui caractérisent les pieds des grands ensembles en banlieue. Alors que les seconds renvoient à des images de la ville ancienne, aujourd'hui très valorisée. Il s'agit des cours des immeubles parisiens d'aujourd'hui. Si ces deux cas de figure apparaissent plutôt extrêmes, ils relèvent cependant d'une même catégorie que l'on nomme les "espaces intermédiaires", autrement dit des espaces censés opérer une articulation entre le logement et la ville. Ces espaces de transition se caractérisent par un statut particulier à la charnière entre la sphère domestique et l'espace public.

11 Les sources que nous avons explorées pour rendre compte de ces images reprennent la diversité présentée précédemment. Dans le premier cas, nous nous sommes appuyés sur les images largement véhiculées par les analystes, les artistes ainsi que par les experts de l'aménagement (architectes, urbanistes...) travaillant à leur réhabilitation. Dans le second cas, nous avons également cherché à rendre compte des images propres au milieu de la conception architecturale, mais nous nous sommes davantage attachés à l'analyse des résultats de notre récente enquête ${ }^{13}$ menée auprès d'habitants résidant à Paris dans des immeubles à cour.

\section{Requalifier un lieu en travaillant sur son image}

Dès les années 1970, le bilan concernant les grands ensembles construits dans la périphérie urbaine est sévère. Désignés comme les lieux de la relégation, ils deviennent très vite synonymes de concentration des handicaps sociaux. La dimension collective, qui était censée représenter leur point fort, est montrée du doigt. Elle est assimilée à un véritable échec. Les portraits brossés, ici ou là, reviennent toujours sur ces écueils en dénonçant des règles sociales qui se délitent, une vie de voisinage qui adopte le mode de la réserve et du repli sur soi avec, en point d'orgue, une dégradation des relations intergénérationnelles qui conduit les « jeunes » à « faire la loi » et à s'emparer des lieux collectifs $^{14}$. C'est un type d'habitat qui est ici remis en cause, même si aujourd'hui le procès porte moins sur la forme bâtie que sur ses espaces interstitiels devenus, pour les politiques de réhabilitation, la représentation la plus criante d'une somme de désorganisations. Aussi, les espaces intermédiaires entre les tours et les barres sont présentés aujourd'hui comme étant «la scène par excellence où s'extériorise la crise urbaine $^{15}$ ». Autrement dit, ce qui avait d'abord été qualifié comme de l'» espace libre » 
tend à être disqualifié sous le terme de "vide». François Béguin ${ }^{16}$, géographe et philosophe, dissèque ce changement de regard en démontrant qu'il s'accompagne non seulement d'un glissement sémantique, que l'auteur résume par les termes «vagues, vides, verts ", mais encore d'une évolution dans la production d'images de ces espaces qui, dans les années 1950, contribue plutôt à donner à la banlieue son caractère «bon enfant " pour devenir, quelques décennies plus tard, des diffuseurs d'une vision de la désolation sociale.

Bien que cette analyse s'étende au-delà des seuls pieds d'immeubles en traitant davantage des abords des grands ensembles, elle nous semble pourtant très pertinente pour expliquer la situation qui vise désormais à requalifier les espaces de proximité. C'est pourquoi, pour éclairer l'image d'aujourd'hui, nous reprendrons les différentes étapes que souligne François Béguin. Ce dernier commence par remarquer que ces vastes étendues ont longtemps été désignées sous les termes de "terrains vagues ", et qu'elles sont, tout d'abord, apparues comme des « terres ouvertes à l'aventure et au bricolage, qui perpétuaient aussi le souvenir des entreprises singulières que la ville dense et continue finissait d'exclure de ses rues ${ }^{17}$ ». Dans la première moitié $d u x^{e}$ siècle, alors que l'habitat de la banlieue n'avait pas encore pris sa forme "normalisée et verticalisée », le terrain vague se présentait comme une respiration, un contrepoids face à l'industrialisation de la banlieue. «Plus fécond, plus accueillant, plus terrestre en un mot que celui mobilisé par l'usine ", il donnait à la banlieue son " côté écailleux [...] qui invitait à considérer le vague comme de l'étendue indéterminée riche de possibles ${ }^{18} »$. Pour nous remémorer cette image, François Béguin puise dans la littérature, le cinéma et s'attarde plus encore sur les photos prises par Robert Doisneau dans la banlieue de Paris entre 1932 et 1945. Elles témoignent d'un large potentiel d'activités : jeux d'enfants, déjeuners sur l'herbe, mais aussi pratique de la chasse et promenade des chèvres... Tout se passe comme si ces espaces révélaient ou réveillaient une démarche volontaire et active de l'habitant. Au contraire, les photos prises par le même artiste, mais dans les années 1980, montre " des espaces vides, inoccupés ${ }^{19}$ » qui, avec d'autres, ont contribué à véhiculer une image où les grands ensembles apparaissent comme "entourés de leur glacis stérile ${ }^{20}$ ». Ici, l'habitant ne semble plus remplir et animer l'espace : «Dans ce cadre, l'homme n'est pas en train de construire un monde à travers une activité, il ne fait que jouer le rôle qu'on lui destine dans un monde conçu par d'autres ${ }^{21}$. " Entre ces deux séries d'instantanés, les années 1950-1960 voient l'émergence des tours et des barres qui s'accaparent ces espaces pour en faire, en théorie, un point fort de leur concept: «Grâce à lui [l'espace libre], l'air, la lumière, la vue s'ouvrent de vastes avenues et le sol devient disponible pour des pelouses, des arbres et des parkings ${ }^{22}$.» De ces trois derniers éléments, les experts contemporains n'y voient plus que l'étalement surdimensionné de vastes nappes de voitures plombant le paysage. Et les médias de se faire l'écho des « actes de sauvagerie » commis sur ces terres. «Fragments d'espaces disjoints et manquant de liaisons suffisamment lisibles avec la ville environnante ${ }^{23}$ ", ces interstices sont stigmatisés en raison de l'indétermination de leur statut et de leur usage, qui, après avoir joué en faveur des habitants, s'imposent comme un facteur aggravant dans leurs conditions de vie et dans la «crise » qu'ils traversent. Aussi, de nombreux projets urbains se fixent-ils pour objectif de résorber ces «vides». Une des solutions imaginées consiste à apposer à ces espaces du désordre une figure fortement structurante et qualifiante socialement : celle de la résidence.

14 La stratégie de la résidentialisation, présentée depuis quelques années comme l'un des remèdes opératoires, repose sur l'idée qu'il est possible de s'opposer efficacement aux 
ferments de la dégradation sociale en intervenant sur la forme des espaces collectifs. Le principe consiste à regrouper ou à découper les immeubles (tours et barres) en " unités résidentielles" par une série de marquages et de limites matérielles (haies, clôtures, barrières, traitement des sols différencié...). Les aménageurs disent avoir choisi cette image de la « résidence » pour ce qu'elle sous-entend dans le changement de valeur de l'habitat et des pratiques qu'elle autorise, telles que "pouvoir recevoir à nouveau et recouvrir la fierté d'habiter ${ }^{24} »$. Il s'agit donc d'interventions qui se fixent pour objectif de « redonner du sens ${ }^{25}$ » à ces espaces en les réaffectant à des petits groupes d'habitants et en les réorganisant de manière à retrouver la composition rassurante de la ville dense avec son réseau de voiries cohérent et un système lisible d'espaces publics. Les aménageurs disent vouloir « sortir de l'espace homogène du terrain vague pour fabriquer des lieux avec des qualités particulières et différentes ${ }^{26}$ ». À une représentation d'un territoire lisse, sans épaisseur, perçu comme un "dramatique nulle part ${ }^{27}$ », ces urbanistes opposent celle d'un territoire organisé sur un principe de différenciation générateur d'usages et de caractères qualifiants.

Il se trouve que, d'après des enquêtes sociologiques récentes, cette nouvelle configuration semble parfois basculer dans l'image négative de la ghettoïsation. C'est ce que relate la sociologue Virginie Véricourt dans son reportage Lieux communs traitant de la réhabilitation récente de la cité Beaumarchais à Montreuil: "Le concept de résidentialisation prête à des distorsions et des manipulations qui conduisent à une ghettoïsation accrue des cités ${ }^{28} »$ et de dénoncer une approche trop formaliste qui ne tiendrait pas compte du contexte social et historique propre à chaque situation. Certains analystes vont plus loin en accusant ces opérations d'être des entreprises de normalisation et de mise sous contrôle d'une population, en ce sens que « la privatisation et l'embellissement des espaces attenant aux logements sont calqués sur les normes de sociabilité des classes moyennes ${ }^{29}$ ». Ainsi, leurs enquêtes tendent, à travers les propos des habitants, à reconsidérer la spécificité de l'organisation sociale de ces grands ensembles : «À un consensus difficilement acquis et longuement mûri dans le temps, succéderait trop brutalement une organisation imposée avec des règles dans lesquelles les habitants ne se reconnaîtraient pas. Le remodelage des formes et le changement des règlements, en modifiant certaines pratiques anciennes et bien ancrées, bouleverseraient l'équilibre global des relations sociales façonné dans le temps au sein d'un territoire donné ${ }^{30}$. » Certains architectes emboîtent d'ailleurs le pas de ces études en fustigeant les opérations de résidentialisation qui, selon eux, consistent à faire de la «pseudo-ville » en négligeant "la manière particulière et polémique avec laquelle les habitants se sont accaparés leur cité ${ }^{31}$ ». Ils proposent des démarches qui reposent sur une autre représentation des grands ensembles, puisqu'ils s'attachent à leur reconnaître des «qualités propres qui tiennent essentiellement aux grands espaces, qu'il s'agisse des espaces verts entre les immeubles ou des zones de parkings en pieds de tours ${ }^{32} »$. De ce fait, les interventions proposées changent de nature et consistent à créer des pôles de services qui cherchent à être le relais d'usages informels déjà bien installés.

Entre cette dernière logique et celle de la résidentialisation, les aménageurs ont à la base une représentation divergente non seulement concernant la potentialité des grands ensembles mais aussi quant à la capacité des individus à la développer. Dans la logique de la résidentialisation, l'habitant, tout désigné pour la vie citadine, se voit désorienté dans cette immensité hostile, et ainsi dépossédé d'une vie sociale. L'aménagement proposé vise alors à lui réinsuffler une urbanité perdue qui lui permette de réinvestir le territoire 
collectif en y exerçant un contrôle et en y éprouvant une responsabilité. Dans l'autre démarche, l'habitant est plutôt considéré comme producteur sur son territoire d'une multitude de pratiques, aussi hostile que puisse paraître cette terre d'accueil. C'est dans ce sens que l'un de ces architectes expose les fondements de son dernier projet de réhabilitation en répercutant les résultats d'une étude qui montre (entre les deux barres d'une cité dans les quartiers Nord d'Amiens) « une quantité d'usages précis du territoire, tels qu'une laverie pour les femmes africaines installée dans un préfabriqué au milieu des arbres, un espace de jeux pour les petits, un terrain de foot pour des ados, un espace de repos sous les arbres au soleil couchant pour les personnes âgées ${ }^{33} \ldots$... Cet inventaire n'est pas sans nous rappeler les premières photos de Robert Doisneau.

Assisterait-on alors à un retournement d'image qui viserait à redonner aux pieds des grands ensembles une épaisseur sociale capable d'instiller une revisitation de nos clichés?

\section{Cultiver l'image d'un lieu}

18 Si les espaces ouverts de la banlieue sont vécus comme un lourd tribut à payer pour les dernières générations d'architectes, il est d'autres formes urbaines qui se révèlent, au contraire, fertiles pour leur imaginaire. En réaction à « l'expérience la plus radicale et anti-urbaine qui ait étée ${ }^{34}$ ", les architectes se sont repris à rêver d'une ville resserrée. C'est ainsi que l'image de la cour s'est réimposée en force dans leur travail de composition. Alors que les interminables étendues de la banlieue, baptisées parfois " cours sans ville ${ }^{35}$ " semblent glacer leur inspiration, les architectes apparaissent, au contraire, transportés par les cours de la ville ancienne qu'ils voient comme des jardins secrets « creusés dans le calme des demeures ${ }^{36}$ ». En témoigne l'ouvrage Paris, côté cours... ${ }^{37}$ qui se fait largement l'écho de cette vision poétique et nostalgique que suscite chez les concepteurs l'évocation de ce type de lieu. Dans des récits très personnels, ils nous livrent les contours d'un espace au fort pouvoir imageant qui les invite à mêler rêve et réalité, expériences sensorielles et imaginaire urbain, souvenirs personnels et mémoire collective, le tout se chargeant d'une forte résonance affective. Certains s'attachent plus particulièrement à traiter de la cour comme d'un lieu intime, «transitoire [qui nous met] à l'abri de l'air agité du boulevard ${ }^{38}$ » et qui accueille en son sein des pratiques sociales dont la ville ne saurait prendre soin. D'autres y discernent des « lieux de mémoire ${ }^{39}$ » qui, par une série de détails pittoresques (gros pavés « Napoléon » aux joints enherbés, murs de meulière protégeant des cours plantées), les renvoient à « un état antérieur de la ville ${ }^{40}$ ». Sorte de parcours initiatique, si elle raconte le passé, elle prépare aussi le regard du futur architecte qui y voit un terrain idéal d'observation des mille et un détails propres à rendre la beauté d'un espace. Par ailleurs, tous soulignent le contraste que l'univers de la cour marque avec la ville dans ce qu'il offre de "caché, secret et réservé ${ }^{41}$ ». Nous pourrions, pour l'illustrer, reprendre les mots de cet architecte qui ne cache pas d'être davantage plongé dans la rêverie que dans la réalité :

Dans les belles pages de l'atlas urbain imaginaire que le promeneur des villes traîne au gré de ses parcours, figure en bonne place le jardin caché : pas la cour réduite, serrée, coincée, mais celle qui le temps d'un porche vous transporte d'une rue dense et sonore vers un jardin calme, éclairé, luxuriant refuge de quelque vie cachée et secrète: lieux dévoilés au hasard d'une rencontre, d'une porte entrouverte ou d'un code détraqué. Certes, cette disposition est un fantasme digne de la ville à la ville ${ }^{42}$. 
Dans ce lot de rêveurs se trouve un trublion, plus terre à terre, qui dénonce précisément le rapport fantasmatique qu'entretiennent les architectes à la cour. Et de penser, dans une logique trop formaliste, que ce type d'espace clos resterait « le microcosme stable et mesuré [capable de réaliser] un contrepoint idéal aux dérèglements de la Babylone moderne ${ }^{43}$ ». Il leur reproche de surinvestir le principe de la cour des faubourgs, qui, dans les activités laborieuses qu'elle accueillait, produisait une "densité sociale où s'accumulaient les temps de travail, du social et de l'intime ${ }^{44} »$. Ces pratiques aujourd'hui révolues, il serait, selon lui, vain de toujours penser que de manière systématique « la cour ferait correspondre l'épaisseur de la ville à l'épaisseur des relations sociales ${ }^{45}$ ».

Il n'en reste pas moins que, depuis une vingtaine d'années, des cours et des jardins sont réapparus dans les nouveaux groupes d'habitation des centres urbains et de la périphérie, qu'ils soient financés par l'Etat ou par des promoteurs privés.

21 S'il ne semble plus faire de doute aujourd'hui que l'immeuble à cour est réinvesti par les responsables de projet (maîtres d'ouvrages, urbanistes, architectes, paysagistes, etc.), qu'en est-il des habitants, de ceux qui, au quotidien, s'y déplacent, y rêvent, y agissent, y font des rencontres? La cour de l'habitant vibre-t-elle d'une même surface imageante que celle du concepteur?

22 C'est dans le but d'apporter des éléments de réponse à ces interrogations que nous sommes partis à la rencontre d'une population logée dans des immeubles à cour récemment construits ${ }^{46}$, situés dans les limites de la capitale. Bien que nous ayons cherché à représenter toutes les catégories socio-économiques de logements, nous avons surtout obtenu des entretiens dans l'habitat social. Cette catégorie est aussi plus largement médiatisée car jugée par les critiques d'architecture souvent plus audacieuse que celle produite par les maîtres d'ouvrage privé. Avant d'envisager le travail d'enquête, il nous a en effet été nécessaire de réaliser un repérage de ces nouvelles constructions implantées dans Paris intra muros. Nous avons donc consulté des revues d'architecture et d'urbanisme mais aussi les informations disponibles à la Préfecture de Paris et dans les fonds documentaires de quelques responsables des projets (architectes, maîtres d'ouvrage public et privé). Les opérations, une fois repérées, faisaient l'objet sur place d'un état des lieux qui permettait de mettre en valeur leurs qualités urbaines et architecturales, spécifiquement en rapport avec les espaces de la cour. Sur la quarantaine d'opérations que nous avons traitées, douze ont été retenues pour conduire une enquête sociologique, la sélection ayant été faite dans le but de représenter des différences architecturales et urbaines, mais aussi sociales et géographiques. Au cours d'entretiens approfondis menés avec une partie des résidents, nous avons cherché à explorer l'étendue des relations qu'un habitant entretient avec son cadre de vie dans le domaine des pratiques mais aussi des représentations. Ce sont celles-ci que nous nous proposons de relater ici.

Les concepteurs, nous l'avons vu, se font les rapporteurs d'une histoire de la cour, souvent très embellie. Chez les habitants, nous avons aussi trouvé la trace de cet imaginaire collectif idéalisé même s'il arrive qu'il soit assombri par des expériences personnelles. C'est ainsi qu'un propriétaire se refuse à appeler l'espace au pied de son immeuble une cour: "J'ai parlé de " cour ", ça m’a échappé car le mot cour est péjoratif pour moi. En effet, les cours que j'ai connues étaient plutôt des cours obscures et désagréables. Appeler cour l'espace qui s'ouvre en dessous ici, c'est péjoratif, car ici c'est un espace clair, il est aéré, il n'est pas entouré de murs ${ }^{47}$." Marqué par les "tristes arrières " qui caractérisaient l'immeuble haussmannien où résidaient ses parents, cet 
habitant nous renvoie à une mémoire peu glorieuse de la cour qui, en cette période, avait cessé d'être un espace de qualité pour ne devenir que le « reste » entre les bâtiments. Des historiens ont d'ailleurs rebaptisé " puits noirs » ces "sinistres courettes parisiennes » que «les propriétaires et architectes, alors peu soucieux de préoccupation sanitaire, avaient désigné, sans vergogne, comme des "puits de lumière" 48 ». Des romanciers comme Zola ont dépeint ces cours comme désespérément infectes, où les prolétaires venaient croupir dans la misère et l'alcoolisme. Cependant, aujourd'hui il n'est plus question pour les architectes de penser ces espaces à ciel ouvert à partir d'une seule fonction de service. Chassant de leurs références cette mémoire noire où les interstices pouvaient être synonymes d'insalubrité dans la ville, ils cherchent dans leurs réalisations à défendre une autre image de la cour: une cour envisagée comme un lieu architecturalement et socialement défini. C'est d'ailleurs dans ce sens que les habitants interprètent en règle générale les intentions des architectes qui ont conçu leur lieu d'habitation : «On sent que l'architecte a eu envie de donner un peu de verdure au milieu de l'immeuble, une respiration et aussi de faire en sorte que ça soit plus convivial, que les gens puissent se parler plus naturellement ${ }^{49}$.»

24 En vivant dans un immeuble à cour, il n'en reste pas moins que les habitants aspirent aussi à retrouver certains traits qui caractérisent l'image romantique qu'ils s'en font, notamment celle du jardin secret. Aussi, ce locataire exprime-t-il sa frustration quant à l'entrée de son immeuble qui donne d'emblée à voir tous les atouts du bâtiment, ce qui pour lui est contraire à la progression attendue : «S'il y avait eu une porte opaque plutôt que cette grille, les gens auraient été d'autant plus surpris de l'intérieur. Il y a des endroits merveilleux dans Paris où on pousse une porte et on passe de la ville au jardin, à la forêt. Là on n'a pas besoin de franchir la grille, on voit tout ${ }^{50}$. » On peut dire que cette idée de la découverte des profondeurs de la ville est largement partagée; nous l'avions vue déjà très prégnante dans la rêverie des architectes. Elle insiste particulièrement sur le dispositif qui prépare à la découverte ; celui-ci semble en effet tout aussi important que ce qu'il autorise à révéler. C'est pourquoi les habitants se montrent sensibles aux qualités de la transition entre la rue et le cœur de l'immeuble, qui, d'une manière générale, ne leur paraissent pas mobiliser dans les constructions contemporaines tous les soins qu'elles méritent.

Dans cet esprit pittoresque, la cour peut être appréciée quand elle se fait l'écho d'un quartier que l'on a particulièrement choisi pour ce qu'il représente de l'âme du Paris d'autrefois. Il en est ainsi pour les habitants de cette opération située au cœur de Ménilmontant, qui voient les espaces à ciel ouvert de leur résidence comme des prolongements fidèles à l'état d'esprit de ce qu'ils sont venus chercher dans ce coin de Paris : «Le fait d'avoir dans la résidence ce passage au milieu, cet escalier, les petits immeubles, les appartements qui sont tous différents, c'est tout à fait l'esprit que l'on trouve ici. Ça fait penser aux anciens escaliers du quartier, à l'idée de ces passages, de ces porches et des jardins un peu secrets que l'on rencontre en se baladant ${ }^{51}$. »

Alternative au Paris d'aujourd'hui mais aussi alternative aux nuisances de la ville, la cour est souvent vécue comme une compensation. Elle l'est d'autant plus quand elle peut offrir un véritable havre de verdure. Et, c'est à partir du moment où le lieu permet la métamorphose de la cour en jardin qu'il semble gagner ses lettres de noblesse : « Il y a beaucoup de cours dans Paris, peut-être moins grandes que celle-ci, mais plus sympas parce qu'ici c'est une cour jeune où la verdure est encore jeune, les arbres tout petits. Dans les immeubles en pierre de taille, les cours ont peut-être cent ans, il y a des arbres, 
des tas de trucs ${ }^{52}$.» La demande de nature dans les cours est largement exprimée par les habitants : "C'est de plus en plus vert et tant mieux car il faut enlever tout le béton ${ }^{53}$." On peut saisir derrière ce souhait le désir paradoxal de trouver la campagne à Paris. Celleci renvoie non seulement à l'idée de nature mais aussi à une échelle différente de celle proposée par la densité urbaine. C'est pourquoi elle se voit définie par ses maisons plus basses, ses ruelles pittoresques, son aspect caché, inattendu, et son caractère communautaire. En opposition à l'idée commune de ville, caractérisée, elle, par de grandes avenues, des immeubles élevés et son anonymat présumé, la campagne à Paris apparait comme une image inversée de cette représentation. C'est d'ailleurs bien ce que nous laisse entendre cet habitant d'un secteur $\mathrm{du} 20^{\mathrm{e}}$ arrondissement récemment reconstruit dans l'esprit faubourien : «Quand on sort du métro, on voit la maison. Je vois la verdure. Plus je me rapproche, plus je m'éloigne de la ville. C'est une transition assez douce. On passe d'un monde à un autre. On arrive vers un lieu de respiration, de parfum, c'est un peu la campagne ${ }^{54}$.»

27 À côté de cette ambiance champêtre, il est des opérations de logements où les matériaux et les dispositifs spatiaux mis en œuvre vont davantage rappeler les attraits d'un paysage et d'un climat méditerranéen. Dans l'opération de la rue Piat, la couleur claire du bâtiment, le relief donné par le tissage des circulations à ciel ouvert, le surplomb sur la ville produisent cet effet : « Je rentre par l'immeuble. J'ouvre la grille, je vois un tout petit peu cette vue sur la ville. Je plonge dans la ville. Je monte l'escalier, tout autour de moi, c'est blanc. J'ai le sentiment d'être dans un village du Sud de peu d'habitants ${ }^{55}$.» On assiste à ce même type d'évocation rue de l'Amiral Mouchez; cette fois elle semble nourrie par l'entrée de l'immeuble qui se distingue d'un hall classique en proposant un vaste porche qui communique directement avec la cour, et également par le départ des ascenseurs et des escaliers qui, à l'air libre, offre une vue sur cette cour: "Quand on rentre dans un immeuble, d'habitude on rentre par un sas; là, il n'y a pas d'entrée, parce que c'est tout ouvert. Et puis après il y a un ascenseur ou l'escalier et, pour le rez-dechaussée, on suit un petit chemin pour arriver dans son appartement. Et lorsqu'on sort de l'ascenseur, hop! on est dehors. C'est agréable. C'est aéré. C'est assez méditerranéen en fait comme esprit ${ }^{56}$. " Quand les cheminements dans les parties communes prolongent le rapport au dehors déjà offert par la cour, l'immeuble semble investi d'images de contrées où le climat se vit de manière sensible, souvent parce qu'il y est agréable. En témoigne ces propos: "C'est vraiment un immeuble comme on fait en Floride où il fait beau toute l'année parce qu'il y a des ouvertures partout ${ }^{57}$.» On saisit ici le poids de la pratique de ces espaces, mais plus encore le rapport sensible qui s'opère avec eux, un rapport générateur de multiples sensations qui s'organisent ensuite en un tout cohérent pour donner l'image de la Méditerranée ou de la Floride. On l'observe encore dans cet autre propos : «Je suis très sensible à l'architecture. Ici je trouve qu'elle est lumineuse ; quand on arrive ici on se sent bien. J'aime bien ce blanc, qui reflète la lumière, j'ai vécu dans les pays méditerranéens pendant dix ans. Ici, je retrouve cette lumière ${ }^{58}$. "

À travers ces projections de paysages, ce sont aussi des modes de sociabilité, une vision des rapports de voisinage qui transparaissent. L'idée de la campagne comme celle d'une ville du Sud portent souvent en elles le projet d'une vie communautaire harmonieuse. C'est pourquoi, on entend souvent, dans leurs propos, les habitants associer la Méditerranée à la convivialité, à la chaleur des relations humaines, et la campagne, à l'esprit villageois. De telles références peuvent être aussi convoquées pour justifier une certaine promiscuité créée par le dispositif de la cour. C'est ce que nous laisse entendre 
cette locataire de la rue des Vignoles : «Moi, ce qui me plait, c'est cette ouverture sur cette cour. C'est ce côté village. Ça rappelle aussi l'Italie, les uns sur les autres, les balcons à l'italienne, les coursives. Il y a de ça ${ }^{59}$. " L'intensité des vis-à-vis semble dans ce cadre davantage productrice d'une valeur communautaire que d'un inconvénient pour la vie urbaine.

Si elle ne parvient pas toujours, comme dans cette opération, à être investie de valeurs collectives, la cour est, d'une manière générale, appréciée pour le sentiment d'espace qu'elle procure. Et c'est dans ce sens qu'elle participe à la revalorisation de l'habitat social, très souvent associé à l'image de la surdensité, comme en témoigne cette habitante : «J'aime bien l'immeuble, parce qu'il y a vraiment de l'espace. Par rapport à un petit immeuble tassé, là on n'est pas les uns sur les autres. C'est pas une grande tour non plus. Ça part à gauche, en haut. Je m'y sens bien ${ }^{60}$. » Le développement des espaces à ciel ouvert comme principe d'agrément participe ainsi pour ces habitants à ne plus considérer leur habitat comme du logement social ordinaire et ainsi à revaloriser leur propre identité résidentielle.

Néanmoins, la cour peut aussi rappeler avec force une position de soumission vis-à-vis d'une autorité et ainsi ramener l'habitant à un statut d'irresponsable et d'assisté. En effet, dans ces groupes d'habitation, il y a très souvent un gardien chargé par les sociétés de gestion de faire respecter un règlement draconien limitant les parties communes à une seule fonction de passage (ni jeux ni fêtes de voisinage ne sont autorisés). Si ce règlement peut être assoupli dans les immeubles où les habitants sont jugés "bons résidents ", il peut aussi être renforcé dans les quartiers difficiles. C'est ainsi que dans des opérations comme celles de la rue de la Goutte d'Or et du boulevard Macdonald, les cours, pourtant prévues pour l'occupation (aménagées de bancs et d'espaces verts), sont rendues inaccessibles à leurs habitants, et deviennent alors des espaces inanimés, sortes de décors surajoutés trahissant la pauvreté ambiante. Aussi, si ces lieux conservent une «belle image ", comme une œuvre doit rester fidèle à son auteur, ils sont peu pratiqués par les habitants et prennent alors pour eux les allures d'une ville-musée inappropriable. C'est dans ce sens que l'on peut s'interroger sur ces opérations de résidentialisation en vogue dans les grands ensembles qui visent à plaquer une image, certes éprouvée dans la ville traditionnelle, mais qui semble faire peu de cas des spécificités du contexte dans lequel elle s'applique.

Que penser en effet d'une manière générale des interventions d'aménagement ou de gestion qui visent à rendre conforme un environnement, à le dégager de toutes ses aspérités? À trop vouloir cultiver l'image, ne la rend-on pas stérile aux yeux des habitants?

\section{NOTES}

1. Fonction que l'on peut lire aujourd'hui comme un prolongement au logement, différencié du domaine public. Mais nous reviendrons plus en détails sur ce statut particulier dans la suite de l'article. 
2. Maïté Clavel, Sociologie urbaine, éditions Economica, Anthropos, Paris, 2002, p. 43.

3. Kevin Lynch, L'Image de la cité, Dunod, Paris, 1976, p. 149 ; traduit de l'anglais, (Mass.), The MIT Press, 1960.

4. Gustave-Nicolas Fischer, Psychologie de l'environnement social, Dunod, Paris, 1997, p. 73.

5. Jacqueline Palmade citée par Jean-Michel Léger dans Derniers Domiciles connus. Enquête sur les nouveaux logements 1970-1990, éditions Créaphis, Paris, 1990, p. 27.

6. L'Évasion domestique. Essai sur les relations d'affectivité au logis, Presses polytechniques et universitaires romandes, Lausanne, 1990, p. 15.

7. L'Image de la cité, Dunod, Paris, 1976 ; id.

8. Id., op. cit., p. 154.

9. Maïté Clavel, op. cit., p. 44.

10. Marc Crunelle, Toucher, audition et odorat en architecture, éditions Scripta, Lanrodec, 2001, p. 88.

11. G.-N. Fischer rappelle, qu'entre autres explications de cette production imaginaire, a été avancée l'idée de projection. C'est à la définition donnée par Anzieu et Martin (1979) qu'il nous renvoie pour définir la projection : le mot projection désigne, en psychologie, le mécanisme par lequel chacun tend à attribuer à autrui des sentiments dont il est en réalité le sujet. Dans G.-N. Fischer, op. cit., p. 183.

12. Op. cit., p. 8.

13. Cette enquête est réalisée dans le cadre d'une recherche financée par le ministère de l'Équipement. Proposée et dirigée par Cristiana Mazzoni, elle s'intitule: Cours et jardins d'aujourd'hui: le renouveau d'espaces aux qualités multiples, Puca, 2003. Cette démarche d'enquête s'inscrit également dans le cadre de notre travail de thèse traitant plus largement des espaces intermédiaires dans l'habitat collectif d'aujourd'hui. Elle avait été initiée par notre mémoire de DEA «Frontières du "chez-soi" et espaces intermédiaires dans l'habitat collectif», École d'architecture Paris-Belleville, juin 2001.

14. Lieux que la presse ou les politiciens aiment souvent à désigner sous les termes de «cages d'escaliers ».

15. Propos de Wanda Diebolt, directrice de l'architecture et du patrimoine au ministère de la Culture, dans le cadre d'un appel d'offres lancé en 2000 totalement consacré aux espaces intermédiaires : Entre privé et public, les rapports de cohabitation et les usages des espaces communs dans les ensemblesrésidentiels, Direction de l'architecture et du patrimoine, 2001, p. 5.

16. Dans « Vagues, vides, verts ", Le Visiteur, $\mathrm{n}^{\circ} 3$, 1997, p. 56-69.

17. Id., op. cit., p. 56.

18. Id., op. cit., p. 60.

19. Id., op. cit., p. 57.

20. Id., op. cit., p. 58.

21. Id., op. cit., p. 59.

22. Id., op. cit., p. 63.

23. Wanda Diebolt, op. cit., p. 5.

24. Philippe Panerai, «Grands ensembles et résidentialisation ", Conférence ACS, 8 avril 2001, Paris.

25. Id., op. cit.

26. Christian Devillers, «Le projet urbain » dans François Cuillier, Les Débats de la ville 1, éditions Confluences, 1998, p. 36.

27. Antoine Grumbach, « La dialectique des contraintes », Le Débat, Paris, Gallimard, août 1994, p. 144.

28. La Huit, 2002. 
29. Claire Levy-Vroelant et al., «Les enjeux de la résidentialisation » dans Appel d'offres : Entre privé et public, les rapports de cohabitation et les usages des espaces communs dans les ensembles résidentiels, Direction de l'architecture et du patrimoine, 2001, p. 31.

30. Id., op. cit., p. 31.

31. Nicolas Michelin, «Comment éviter de passer de la barre à l'îlot? ", AMC, $\mathrm{n}^{\circ}$ 127, septembre 2002, p. 38.

32. Id., op. cit., p. 38.

33. Id., op. cit., p. 38.

34. Jacques Lucan, «Ouvert/fermé », dans Pierre Gangnet (dir.), Paris, côté cours. La ville derrière la ville, éditions du Pavillon de l'Arsenal, Picard, Paris, 1998, p. 176.

35. Alexandre Chemetoff, « Le paysage des cours », dans Pierre Gangnet (dir.), op. cit., p. 32.

36. Jean-Pierre Le Dantec, « Madame Ariane, 2e cour à gauche », dans Pierre Gangnet (dir.), op. cit., p. 15.

37. Pierre Gangnet (dir.), Paris, côté cours. La ville derrière la ville, éditions du Pavillon de l'Arsenal, Paris, Picard, 1998.

38. Jean-Pierre Le Dantec, op. cit., p. 19.

39. Alexandre Chemetoff, op. cit., p. 32.

40. Id., op. cit., p. 29.

41. Id., op. cit., p. 25.

42. François Leclerq, «Éloge de l'épaisseur », dans Pierre Gangnet (dir.), op. cit., p. 189.

43. Dominique Lyon, « Ceci n'est pas une cour », dans Pierre Gangnet (dir.), op. cit., p. 182.

44. Id., op. cit., p. 184.

45. Id., op. cit., p. 182.

46. Opérations de logements construites entre les années 1990 et 2000.

47. Habitant du 22 rue Wurzt, Paris 13e, opération réalisée pour la RIVP par Nicolas Soulier et Patrick Céleste en 1989.

48. Roger-Henri Guerrand, "Dans l'enfer du puits noir », dans Pierre Gangnet (dir.), op. cit., p. 126.

49. Habitant du 62-68 rue de l'Amiral Mouchez, Paris 14e. Opération réalisée pour la SGIM par Michel Kagan en 2000.

50. Habitant du 27 rue de la Réunion, Paris 20e. Opération réalisée pour le ministère des Postes et Télécommunications par Michel Rémon en 1993.

51. Habitante du 25-29 rue du Retrait, Paris 20e. Opération réalisée pour la RIVP par Anthony Béchu en 1998.

52. Habitant du 62-68 rue de l'Amiral Mouchez, Paris 14e. Opération réalisée pour la SGIM par Michel Kagan en 2000.

53. Ibid.

54. Habitant du 15 rue Robineau, Zac des Amandiers, Paris 20e. Opération réalisée pour la RIVP par François Laisney en 1999.

55. Habitant du 45 rue Piat, Paris 20e. Opération réalisée pour la RIVP par Catherine Furet en 1995.

56. Habitant du 62-68 rue de l'Amiral Mouchez, Paris 14e. Opération réalisée pour la SGIM par Michel Kagan en 2000.

57. Ibid.

58. Habitant du 25-29 rue du Retrait, Paris 20e. Opération réalisée pour la RIVP par Anthony Béchu en 1998.

59. Habitante du 56-60 rue des Vignoles, Paris 20e. Opération réalisée pour la SIEMP par Édith Girard en 1997.

60. Habitante du 27 rue de la Réunion, Paris 20ème. Opération réalisée pour le ministère des Postes et Télécommunications par Michel Rémon en 1993. 